J. Lake Sci. (湖泊科学), $2007, \mathbf{1 9 ( 6 ) : 7 1 0 - 7 1 7 ~}$

http://www. jlakes.org. E-mail:jlakes@ niglas.ac.cn

(c) 2007 by Journal of Lake Sciences

\title{
在土槽尺度下的硝态氮迁移模拟研究”
}

\author{
梁 威 ${ }^{1,2}$, 刘 凌 ${ }^{1,2}$, 潘 沛 ${ }^{1,2}$
}

( 1 : 河海大学水文水资源与水利工程科学国家重点实验室,南京 210098)

$(2$ :河海大学水资源环境学院水文系,南京 210098)

摘 要:农业非点源污染研究是当前环境问题研究领域的重点和难点. 目前而言土槽尺度下非点源模型的研究较少. 利 用大型土槽和人工降雨装置来模拟无植被坡地单次降雨过程地表径流中硝态氮迁移过程. 在此基础上, 适当选取 ANSWERS - 2000 的子模块建立起具有一定机理的适用模型, 来研究土槽尺度下无植被坡地单次降雨过程地表径流中硝态 氮迁移过程. 经过三次实验验证, 模拟的径流总量误差在 4\%-15\% 之间, $C P_{A}^{\prime}$ 在 $0.7-12$ 之间; 模拟的硝态氮总量误差 的绝对值在 9\%-17\% 之间, $C P_{A}^{\prime}$ 在 $0.6-1.1$ 之间. 结果显示降雨径流、硝态氮浓度模拟结果与实际观测值有较好的一 致性,模型模拟效果较好,模型可用于该尺度下硝态氮迁移量计算,具有一定的研究参考价值.

关键词: 农业非点源污染; 土槽尺度; 降雨径流; 硝态氮; ANSWERS - 2000 模型; GREEN-AMPT 下渗模型; 迁移模拟

\section{Simulation of nitrate-nitrogen transfer in trough scale}

LIANG Wei ${ }^{1,2}$, LIU Ling ${ }^{1,2} \&$ PAN Pei ${ }^{1,2}$

(1:State Key Laboratory of Hydrology-Water Resources and Hydraulic Engineering, Hohai University, Nanjing $210098, P$. R. China)

(2: College of Water Resources and Environment, Hohai University, Nanjing 210098, P. R. China)

Abstract: Nowadays, research on non-point source pollution which comes from agricultural activities has become a hot-point and difficult-point issue in the area of environmental problem. But currently few researches have been made on non-point source pollution in trough scale. By using big soil trough and artificial rainfall equipment to simulate nature precipitation and flow in uncovered sloping field, a physically-based model based on ANSWERS - 2000 and recombined submodules of ANSWERS - 2000 is developed to study transfer rule of dissolved nutrient ( Nitrate-Nitrogen) in the soil trough during single precipitation. The model had been validated three times. Total runoff errors were between $4 \%-15 \%$ and $C P_{A}^{\prime}$ were between $0.7-12$. Accordingly, total Nitrate-Nitrogen errors were between 9\%$17 \%$ and $C P_{A}^{\prime}$ were between 0.6-1.1. Results showed that the simulation value of runoff and Nitrate-Nitrogen concentration basically accords with the observed value. The model can be used to calculate the transporting amount of Nitrate-Nitrogen in trough scale during single precipitation and had some reference values for future research.

Keywords: Agricultural non-point source pollution; trough scale; precipitation runoff; Nitrate-Nitrogen; ANSWERS - 2000 Model; GREEN-AMPT infiltration model; transfer simulation

近年来, 随着人类经济社会活动的加剧, 人类对工业废水和生活污水等点源污染的治理能力不断提高, 但水环境问题依然严峻, 严重威胁人类的生存和发展. 造成水环境问题的原因除了点源污染, 更重要的是 各种非点源污染, 其中农业非点源污染最易构成水体环境问题. 农业非点源污染是指以降雨为载体, 在降 雨的冲击和淋溶作用下, 通过产流和汇流过程将农田和畜牧用地中的污染物质( 包括土壤颗粒、土壤有机 物、化肥、农药、有机肥料等) 携人受纳水体而引起的水质污染 ${ }^{[1]}$.

* 国家自然科学基金重点项目(50239030)资助. 2006-11-28 收稿;2007-01-17 收修改稿. 梁威,男,1981 年生, 硕士研究生;E-mail: Levin_lw@ hhu.edu.cn. 
正确理解非点源污染物的迁移规律和准确评估非点源污染物的迁移数量是一个世界性的难题. 目前, 非点源模型研究主要有两类方法. 一是统计模型 ${ }^{[2,3]}$, 这主要是污染负荷与其影响因子间建立相关关系, 这 类模型对数据的要求不高, 有一定的精度, 实用性较强, 但它难以描述污染物质的迁移规律, 因而其深人研 究受到限制. 另一种是具有一定机理的模型, 随着对非点源污染理化性质研究的深人以及模拟技术的提 高, 建立一定机理基础的模型逐渐成熟,其应用越来越广. 但是该模型也同样存在缺陷,主要是模型参数繁 多,精度不够理想,数据资料要求较高等. 这类模型比较著名的有 SWMM、ARM、EPIC、ANSWERS、SWAT、 CREAMS 等 ${ }^{[4-9]}$.

目前, 机理模型的研究和运用主要在农田小区或流域. 例如, CREAMS 模型的空间尺度为农田尺度, ANSWERS、SWAT 模型的空间尺度为流域等 ${ }^{[10]}$. 很少有人在土槽尺度下进行建模研究, 在室内土槽尺度下 进行非点源机理的研究, 能够克服野外实验存在实验地不易选定, 影响因素难以控制, 费用较高等的缺陷, 具备实验周期短, 可控程度较高的特点, 是先期进行研究非点源迁移转化规律机理的有益尝试. 本文利用 ANSWERS 模型原理,选取子模块, 建立小型适用模型, 研究该尺度下的硝态氮无植被条件下随地表径流的 迁移过程. 所研究的目的首先是分类和细化研究非点源问题, 其次试图建立适用于该尺度下的非点源迁移 模型.

\section{1 模型选取与建立}

ANSWERS (Areal Non-point Source Watershed Environment Response Simulation) 模型最早由 Huggins 和 Monke 在 1966 年提出, 当时只包括地表水文模块. 1980 年 Beasley 等加人侵蚀和泥沙运输模块, 随后加人

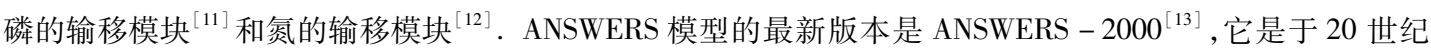
90 年代中期开发的连续性模拟模型.

本文所研究的是以 ANSWERS - 2000 模型为基础, 选取水文模块和溶解态污染物质迁移模块, 建立小 型适用模型, 模拟无植被土槽坡地单次降雨径流以及硝态氮输移过程并与观测结果进行比较.

\section{1 水文模块}

水文模块由下渗、波面汇流所组成. 考虑单次降雨持续时间较短, 土槽坡面较为平整, 槽内基本无植被 生长的情况,所以不考虑蒸发、截流、沟道汇流、植被作用等因素影响.

1.1 .1 下渗过程 土壤的下渗过程采用 GREEN-AMPT 下渗公式 ${ }^{[14]}$.

$$
K s \times t=F-N s \ln \left(1+\frac{F}{N s}\right)
$$

式中, $K s$ 为饱和水力传导度 $(\mathrm{cm} / \mathrm{min}) ; N s$ 为有效基模势 $(\mathrm{cm}) ; t$ 为累积时间 $(\mathrm{min}) ; F$ 为累积下渗量 $(\mathrm{cm})$. GREEN-AMPT 公式是一个具有明确物理意义的饱和下渗公式, 它较好应用于恒定雨强下的地表有积水条 件的下渗及产流计算. 但是一般情况下,雨强是随时间变化的,同时地表也不一定存在积水. Mein ${ }^{[18]}$ 等曾 作过改进,详细过程如下: 对连续时变雨强划分时段,在某一时段内认为该雨强是均匀的,同时把每个时段 都转化为地表有积水条件下的下渗,从而利用 GREEN-AMPT 下渗公式计算下渗量和产流量.

步骤如下 (我们所取的降雨每时段为 $1 \mathrm{~min}$ ):

当 $i=1,2,3 \cdots \cdots$ ( $i$ 为时段计数), 在时段初, 累积下渗量为 $F_{i-1}$. 可由下公式计算时段初的下渗率.

$$
f_{i-1}=K s\left(1+\frac{N s}{F_{i-1}}\right)
$$

式中, $f_{i-1}$ 为时段初对应 $F_{i-1}$ 的土壤下渗率 $(\mathrm{cm} / \mathrm{min}) ; F_{i-1}$ 为时段初土壤累积下渗量 $(\mathrm{cm})$.

到时段末, 由于不知道在这一时段实际下渗量为多少, 所以无法保证整个下渗过程是在地表有积水情 况下发生, 因此无法直接用公式 (1). 可以假设这一时段的所有降雨都渗入地下, 则时段末累积下渗量 $F_{k}$ 为:

$$
F_{k}=F_{i-1}+R_{i} \times \Delta t
$$

式中, $R_{i}$ 为 $i$ 时段雨强 $(\mathrm{cm} / \mathrm{min}) ; \Delta t$ 为时段间隔, $\Delta t=t_{i}-t_{i-1}$, 本次计算为 $1 \mathrm{~min}$.

此时,把所求得的累积下渗量 $F_{k}$ 代人 $(2)$, 可得时段末下渗率 $f_{k}$.

(1) 当 $R_{i}<f_{i-1}, R_{i}<f_{k}$ 时: 该时段降雨全部渗人地下, 实际下渗量 $F_{i}=F_{k}$. 该时段地表无积水, 为了能连 
续使用有积水条件下的 GREEN-AMPT 下渗公式, 可做如下转化. 认为该时段 $R(i) \times \Delta t$ 是在有积水条件下 的下渗量. 把 $F_{i-1} 、 F_{i}$ 代人公式 (1) 各自求得的时间相减, 可得该下渗量在积水条件下的下渗时间为 $t_{i}-$ $t_{i-1}$, 产流量为零.

(2) 当 $R_{i}<f_{i-1}, R_{i}>f_{k}$ 时: 由此可知,在该时段某时刻起,地表开始有积水. 由如下公式 ${ }^{[15]}$ 可求得该时 刻 $T_{p}$ :

$$
\begin{aligned}
F_{p} & =\frac{N s}{\frac{R(i)}{K s}-1} \\
T_{p} & =\frac{F_{p}}{R(i)}
\end{aligned}
$$

式中, $F_{p}$ 是当 $R_{i}=f$ (土壤下渗率) 时的土壤累积下渗量.

所以在这一时段,地表没有积水的时间为 $T_{p}-t_{i-1}$, 假设地表有积水时则下渗时间为 $T_{p}^{\prime}-t_{i-1}$, 其中 $T_{p}^{\prime}$ 是 $F_{p}$ 代人 (1) 所计算的时间; $T_{p}$ 时刻后的地表积水时间为 $t_{i-1}+\Delta t-T_{p}$. 因此这一时段在地表有积水条件 下的时间为 $T_{p}^{\prime}-t_{i-1}+\left(t_{i-1}+\Delta t-T_{p}\right)$. 产流量 $Q_{i}$ 为

$$
Q_{i}=R_{i} \Delta t-\left(F_{i}-F_{i-1}\right)
$$

其中, $F_{i-1}$ 为时段初累积下渗量 $; F_{i}$ 为时段末累积下渗量, 可由地表积水条件下累积时间求出.

(3) 当 $R_{i}>f_{i-1}, R_{i}>f_{k}$ 时: 这一时段完全在地表有积水条件下下渗,则符合 GREEN-AMPT 公式的计算要 求,因此 $\Delta t$ 即为这一时段在地表有积水条件下的下渗时间. 产流量 $Q_{i}$ 计算采用式 (6).

这样,可以把要求在积水条件下的 GREEN-AMPT 下渗公式可应用于任何雨强下的下渗和产流计算.

1.1 .2 坡面汇流 坡面水流运动十分复杂, 一般可用运动波理论、完整圣维南方程描述. 由于运动波理论在 大多数情况下可以很好地描述坡面流运动过程, 而且计算简单. 因此本文采用运动波方程和曼宁公式来描 述坡面水流运动, 水力半径近似等于坡面水深. 如下:

$$
\begin{aligned}
& \frac{\partial q}{\partial x}+\frac{\partial h}{\partial t}=i \\
& v=\frac{1}{n} h^{2 / 3} S_{0}^{1 / 2}
\end{aligned}
$$

式中, $q$ 为土槽水流的单宽流量 $\left(\mathrm{m}^{2} / \mathrm{s}\right) ; h$ 为坡面水深 $(\mathrm{m}) ; i$ 为单位面积上的降水率 $(\mathrm{m} / \mathrm{s}) ; x$ 为自坡顶的 距离 $(\mathrm{m}) ; n$ 为曼宁糙率; $S_{0}$ 为坡度比降.

方程 (7) 经离散化后与 (8) 联用,采用一阶显式迎风格式可求的坡面水流在出口段面的流量过程.

\section{2 溶解态污染物质迁移模型}

溶解态污染物质迁移模型 ${ }^{[15]}$ 基于质量守恒定律, 有一定的机理性. 模型假设污染物全部集中在表层 土中, 即能与降落到表面的雨水能充分的作用. 由此提出土壤的有效交互深度这一概念, 一般认为有效交 互深度为 $1 \mathrm{~cm}^{[15]}$.

在土壤的有效交互深度范围内, 土壤与水充分的作用, 假设污染物质在土壤中和水中的平衡浓度存在 一定的比例关系, 如下 ${ }^{[16]}$ :

$$
K_{n u t}=\frac{C_{s}}{C_{w}}
$$

其中, $C_{s}$ 为土壤中的污染物质浓度; $C_{w}$ 为水体中的污染物质的浓度.

设 $Z$ 是土壤中污染物质含量, 则 $Z$ 在土壤中变化率即为水中带走的污染物质的量:

$$
-\mathrm{d} z=C_{w} w \mathrm{~d} t
$$

式中, $w$ 为有效降雨量, 即除去蒸发、截留后的净雨量.

当土壤饱和时, $Z$ 可认为是土壤水中的污染物质和土壤颗粒污染物质之和:

$$
Z=C_{w} p+C_{s}(1-p) \rho_{s}
$$

其中, $p$ 为土壤孔隙率, $\rho_{s}$ 为土壤容重, 取 $2.65 \mathrm{~g} / \mathrm{cm}^{3}$. 由式 $(9) 、(10) 、(11)$ 推导可得, $Z$ 在降雨径流作用下 随时间的变化方程为: 


$$
Z=Z_{0} \exp \left[-\left(\frac{(Q+f+S) \Delta t}{p+2.65(1-p) K_{n u t}}\right)\right]
$$

式中, $Z_{o}$ 为初始土壤污染物质量; $Q$ 为单位面积流量 $(\mathrm{cm} / \mathrm{min}) ; f$ 为下渗率 $(\mathrm{cm} / \mathrm{min}) ; S$ 为滞水率 $(\mathrm{cm} /$ $\min ) ; \Delta t$ 为时间间隔, 本次为 $1 \mathrm{~min}$.

$Z_{0}, Z$ 可用在土壤中的浓度与土壤质量的乘积表示:

$$
\begin{aligned}
& Z_{0}=C_{i n i t(1-p) \rho_{s}} \\
& Z=C_{a v}(1-P) \rho_{s}
\end{aligned}
$$

式中, $C_{i n i t}$ 为土壤中污染物质的初始浓度. $C_{a v}$ 为土壤中可利用的污染物质的浓度.

当水与土壤污染物质充分作用时, 土壤中 $C_{a v}$ 浓度的污染物质被释放分为水中 $C_{w}$ 浓度的污染物质和在 土壤中剩余 $C_{s}$ 浓度的污染物质. 设 $\beta$ 为单位水体泥沙含量, $V$ 为单位水体体积,因此:

$$
C_{a v} \beta=C_{w} V+C_{s} \beta
$$

则由式(9)、(15), 且 $V=1$, 可得到径流中的溶解态污染物质的浓度 $C_{w}$, 如下:

$$
C_{w}=\frac{C_{a u} \beta}{1+K_{n u} \beta}
$$

$\beta$ 又被称为萃取系数, 与降雨径流、污染物质的溶解性和吸附性等有关. 它的取值见文献 $[15]$.

不同污染物质的分配系数 $K_{n u t}$ 是不同的, 硝氮态氮分配系数 $K_{n u t}$ 为:

$$
\mathrm{NO}_{3}^{-}-\mathrm{N} \text { (硝态氮) }: K_{n u t}=0
$$

\section{2 实验方案设计}

\section{1 实验装置}

实验主要利用河海大学水资源国家重点实验室的大型可变坡土槽, 土槽长 $12 \mathrm{~m}$, 宽 $1.5 \times 2 \mathrm{~m}$, 高 1.5 $m$, 两个土槽并列. 实验设施由人工降雨模拟装置系统、试验土槽系统、水土取样系统、土水势监测系统、雨 量监测系统、土槽驱动及降雨控制系统、测流系统组成. 人工降雨装置由供水与降雨器两个部分组成. 供水 部分采用屋顶水箱供水, 事先需储存自来水; 降雨器采用莲蓬头式喷头酒雨, 其制造和应用均较简单, 缺点 是酒雨强度分布难以控制, 容易造成降雨分布不均匀.

\section{2 实验具体过程}

在不同雨强下 (每次降雨持续时间大约为 $90 \mathrm{~min}$ ), 对流量、水质进行同步观测, 具体做法如下: 雨强由 放置于土槽上的雨量计测得, 在土槽面上均匀放置多个雨量计, 所得雨强数据取平均值计算. 流量用定容 器血(量筒) 测量, 在测流量的同时, 收集水样准备测定其硝态氮含量. 从而得到硝态氮浓度随时间的变化 过程线.

\section{3 采样和样品测定方法}

实验共进行了 3 次,分别为 2005 年 8 月 29 日,2005 年 10 月 8 日, 2005 年 12 月 5 日. 雨强和坡度由计 算机控制, 流量测量和水样采集同步进行(第 $2 、 3$ 次多测了几次流量), 约每 $5 \mathrm{~min}$ 或 $10 \mathrm{~min}$ 一次, 共获得 3 组数据 (图 $1-6$ ). 硝态氮测定采用酚二磺酸分光光度法, 土壤颗粒分析用吸管法, 有机质采用水合热重铬 酸钾氧化 - 比色法等.

\section{4 实验要点说明}

雨强的设计: 首先雨强不能太小, 因为采用莲蓬头式喷头酒雨需要一定的压力, 否则, 喷头酒水将极不 均匀; 其次, 雨强变化幅度不能太大, 因为时间尺度较小, 短时间变化幅度很大的雨强不仅在自然界中少见, 也不利于计算结果的分析. 因此降雨应该尽量接近天然降雨,同时注意降雨分布的均匀性.

土槽中土壤情况: 土壤取自南京江心州长江冲积母质发育的河流砂土土壤, 土槽从 2004 年 8 月初开始 装土, 每 $5 \mathrm{~cm}$ 为一个填土层, 每天根据测量的土壤含水率调整称土重量, 确保 $1.40 \mathrm{~g} / \mathrm{cm}^{3}$ 的干密度的标准, 整个土层厚度在 $1.3 \mathrm{~m}$ 左右. 至 8 月底装土完毕, 共用 $90.7 \mathrm{t}$ 土. 土槽中砂粒土壤下渗性较好, 因此短历时 降雨过程中,基本不存在地下水自由水面.

测流和取样: 在土槽下方有漏斗状的雨水汇集装置, 下部用一软管引出, 在软管另一端用量筒接水测流. 
实验的间隔: 三次降雨的间隔基本都在 2 个月以上, 保证每次降雨后土槽恢复到自然状态, 三次实验较 为独立. 因此,前一次实验对下一次实验的土壤含水量无影响.

每次降雨实验前后, 都要测土壤含水量和土壤硝态氮浓度, 土样采集注重均匀性, 把土槽分为上、中、下三 个部分, 分别从这三部分表层采集土样, 最后三部分土样的硝态氮浓度加权平均作为土槽土壤硝态氮浓度.

\section{3 模型验证和结果分析}

\section{1 模型参数的确定}

模型中的大部分参数可由实验获取. 曼宁䊁率根据水力学资料取 0.035 . 土壤初始硝态氮浓度分别为: $9.98 \mathrm{mg} / \mathrm{L}, 8.44 \mathrm{mg} / \mathrm{L}, 8.39 \mathrm{mg} / \mathrm{L}$. 其它参数见表 1 .

表 1 模型参数

Tab. 1 Parameters of Model

\begin{tabular}{|c|c|c|c|c|c|}
\hline 粘粒含量 $(\%)$ & 分砂含量 $(\%)$ & 砂粒含量 $(\%)$ & 土壤干容重 ( & $\left(\mathrm{cm}^{3}\right)$ 土壤孔隙度 $(\%)$ & 有机质含量 $(\%)$ (3 次 $\left.{ }^{*}\right)$ \\
\hline 7.1 & 16.4 & 76.5 & 1.42 & 48.3 & $43.1 / 41.2 / 40.1$ \\
\hline 田间含水量 $(\%)$ & \multicolumn{3}{|c|}{ 调萎含水量 $(\%) \begin{array}{l}\text { 土壤前期含水量 } \\
(\%)\left(3 \text { 次 }^{*}\right)\end{array}$} & 砾石 $(>2 \mathrm{~mm})(\%)$ & 坡度 $\left({ }^{\circ}\right)\left(3\right.$ 次 $\left.{ }^{*}\right)$ \\
\hline 35 & \multicolumn{3}{|c|}{.2} & 10 & $5 / 3.5 / 3$ \\
\hline
\end{tabular}

* 三次降雨所取的参数值.

\section{2 模型验证}

模型验证的目的是确认模型的正确性、合理性. Heatwole 等认为 ${ }^{[17]}$,一个好的水文模型,如果其参数通 过实地施测获得或经过率定, 那么它的误差应该在 $200 \%$ 以内. 模拟值与实测值系统内部误差可用 $C P_{A}^{\prime}[20]$ 来表示:

$$
C P_{A}^{\prime}=\frac{\sum_{i=1}^{n}(s(i)-O(i))^{2}}{\sum_{i=1}^{n}\left(O(i)-O_{\text {avg }}\right)^{2}}
$$

式中: $O(i)$ 是第 $i$ 个实测值, $O$ avg 是实测值的平均值, $S(i)$ 是第 $i$ 个模拟值, $n$ 是实测值的个数.

$C P_{A}^{\prime}$ 反映系统内部的偏差情况. 当 $C P_{A}^{\prime}$ 值接近 0 , 说明模拟值越接近实测值; 反之, 说明模拟值与实测值 相差越大. Montas ${ }^{[19]}$ 等在实验中用这个值来分析了预测峰值流量与实测峰值流量、预测洪峰时间与实测洪峰 时间和预测径流总量与实测径流总量之间的关系. 其 $C P_{A}^{\prime}$ 值最大为 22.81 , 最小为 0.32 , 平均为 1.84 .

三次模拟的结果见表 2 、表 3. 总量误差和 $C P_{A}^{\prime}$ 分别从总体误差和系统内部误差两个不同方面很好的 反映了模拟与实测的相差程度.

表 2 径流模拟验证

Tab. 2 Validation of runoff simulation

\begin{tabular}{cccccc}
\hline 场次 & 雨量 $(\mathrm{mm})$ & 实测径流总量 $\left(\mathrm{m}^{3}\right)$ & 模拟径流总量 $\left(\mathrm{m}^{3}\right)$ & 总量误差 $(\%)$ & $C P_{A}^{\prime}$ \\
\hline 1 & 111.62 & 1.99 & 1.70 & 14.7 & 0.73 \\
2 & 144.97 & 2.29 & 2.15 & 6.44 & 0.31 \\
3 & 66.91 & 0.93 & 0.89 & 4.03 & 11.76 \\
\hline
\end{tabular}

表 3 硝态氮模拟验证

Tab. 3 Validation of Nitrate-Nitrogen simulation

\begin{tabular}{ccccc}
\hline 场次 & 实测 $\mathrm{NO}_{3}-\mathrm{N}$ 总量 $(\mathrm{g})$ & 模拟 $\mathrm{NO}_{3}-\mathrm{N}$ 总量 $(\mathrm{g})$ & 总量误差 $(\%)$ & $C P_{A}^{\prime}$ \\
\hline 1 & 3.63 & 3.27 & 9.92 & 1.04 \\
2 & 2.49 & 2.25 & 9.6 & 0.67 \\
3 & 0.65 & 0.76 & -16.9 & 0.76 \\
\hline
\end{tabular}




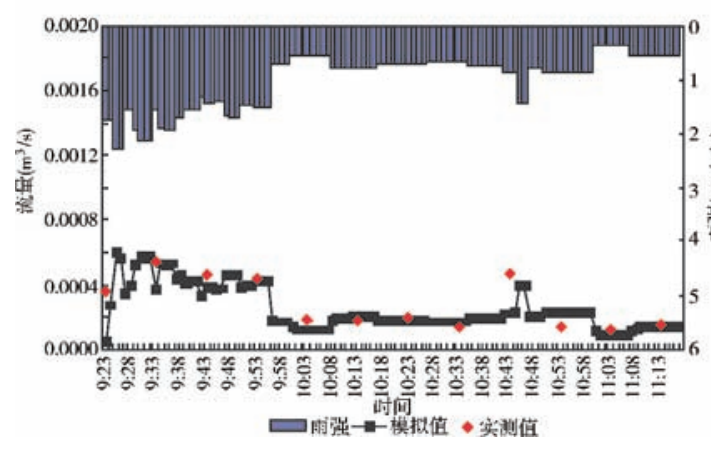

图 1 第一次降雨实测和计算流量过程比较

Fig. 1 Measuring and calculating value of runoff in the first precipitation

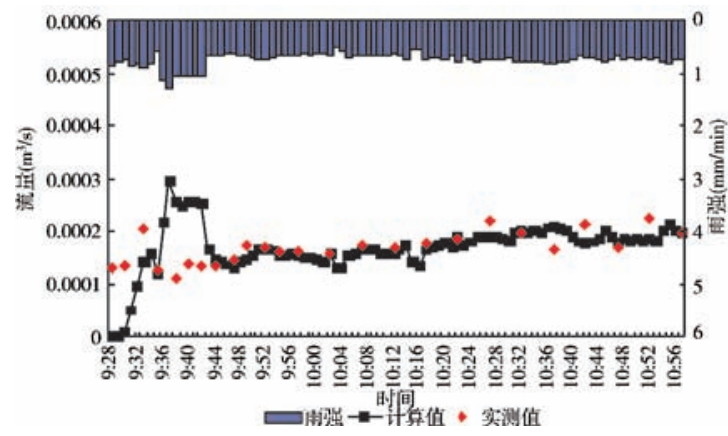

图 3 第三次降雨实测和计算流量过程比较

Fig. 3 Measuring and calculating value of runoff in the third precipitation

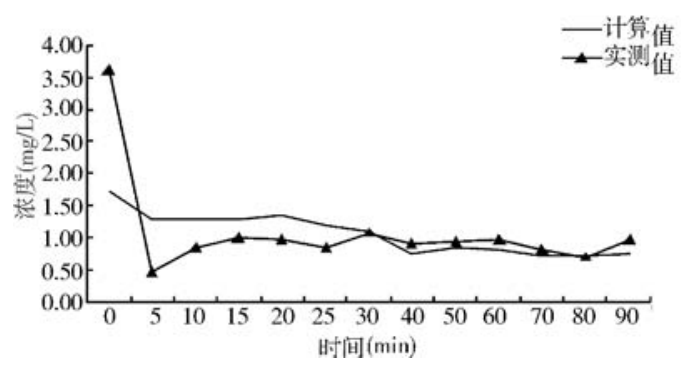

图 5 第二次降雨实测和计算 $\mathrm{NO}_{3}^{-}-\mathrm{N}$ 浓度比较

Fig. 5 Measuring and calculating value of NitrateNitrogen in the second precipitation

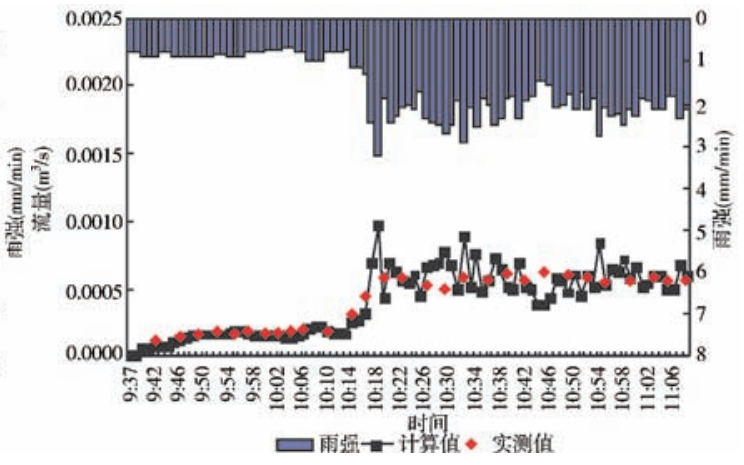

图 2 第二次降雨实测和计算流量过程比较

Fig. 2 Measuring and calculating value of runoff in the second precipitation

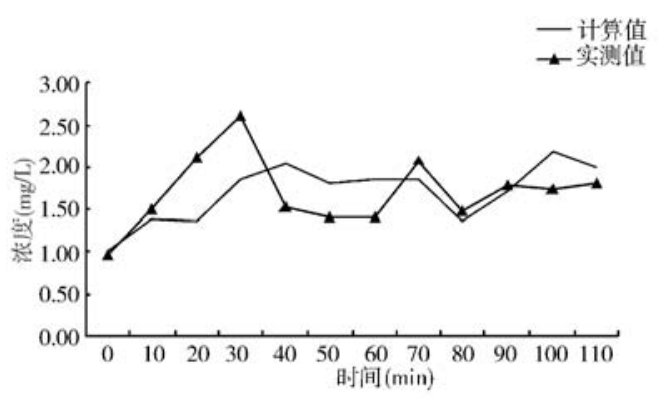

图 4 第一次降雨实测和计算 $\mathrm{NO}_{3}^{-}-\mathrm{N}$ 浓度比较

Fig. 4 Measuring and calculating value of Nitrate-

Nitrogen in the first precipitation $\mathrm{NO}_{3}^{-}-\mathrm{N}$

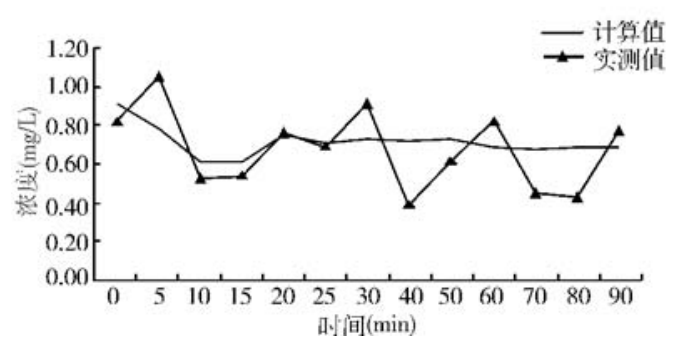

图 6 第三次降雨实测和计算 $\mathrm{NO}_{3}^{-}-\mathrm{N}$ 浓度比较

Fig. 6 Measuring and calculating value of NitrateNitrogen in the third precipitation

\section{3 结果分析}

径流总量误差在 4\%-15\% 之间, $C P_{A}^{\prime}$ 在 0.7-12 之间,与实测相比模拟总量偏小,分析认为这与土壤 下渗能力估计偏大、或曼宁䊁率偏大等有关 (图 $1-3$ 、表 2). 土壤下渗能力由土壤的理化性质决定, 因此这 也与实验的精度和土壤取样点的均匀性有关; 而曼宁䊁率根据有关资料而定, 因此会存在一定的误差. 硝 态氮总量绝对值误差在 9\%-17\% 之间, $C P_{A}^{\prime}$ 在 0.6-1.1 之间,前两场降雨模拟总量小于实测总量,第三场 
降雨模拟总量大于实测总量 (图 4-6, 表 3). 分析认为, 径流模拟的偏小会影响硝态氮模拟总量, 同时也与 初始土壤硝态氮浓度的准确确定和 $\beta$ 等参数有关. 而第三场降雨的硝态氮实测浓度变化过程线变化过于 剧烈, 可能与实验精度有关, 导致较大误差. 另外, 从图中可以看出, 在 $90 \mathrm{~min}$ 内硝态氮浓度随时间并不是 一直呈下降趋势而是呈锯齿状的变动, 同时与出口断面流量过程线变动并不一致. 出口断面流量和硝态氮 浓度的关系是非线性的, 而所建模型能很好地反映这种关系, 说明所建模型的机理一定程度上正确解释了 这种规律性. 总之, 本文所建的适用模型对于土槽尺度下无植被坡地单次降雨过程地表径流中硝态氮迁移 有较好的模拟能力, 具有一定的研究参考价值.

\section{4 结论}

本文所建的模型是适当选取 ANSWERS - 2000 子模块组合而成,具有一定的机理性,适用于模拟土槽 尺度下无植被坡地单次降雨过程地表径流中硝态氮迁移. 模型物理意义清晰, 结构清晰, 参数明确易测, 有 一定的研究参考价值.

模型是在较为理想化条件下所建,因此,对于复杂条件下如何改进需要研究,污染物迁移模块也需要进 一步的改进. 另外本文所述的模型只是模拟无植被坡地溶解态硝态氮随时间的变化规律, 有关其它污染物 的迁移规律、污染物间的转化模块、侵蚀泥沙模块和随泥沙携带的污染物质的迁移模块, 以及不同地表条件 下的坡地污染物质的迁移, 土槽研究结论和农田小区或流域尺度研究结论, 在不同尺度下如何进行结合等 均需要在今后进一步深人研究.

用人工降雨装置和土槽进行研究,有受外界环境影响小、可控性更大等的优点. 但是由于土槽(可看作 是一个小单元) 很小, 敏感性较高, 实验条件的微小改变可能对实验结果产生较大的影响. 因此, 实验精度 的控制也是能否取得研究成果的关键点.

\section{5 参考文献}

１1］庄咏涛,李怀恩. 农业非点源污染模型浅析. 西北水资源和水工程,2001,12(4):12 - 16 .

[2] Whipple W, Hunter J V. Nonpoint sources and planning for water pollution control. Water Pollution Control Federation, 1977, 49:15-23.

[3] 李定强, 王继增等. 广东省东江流域典型小流域非点源污染物流失规律研究. 土壤侵蚀与水土保持 学报, $1998,4(3): 12-18$.

[4] Huber W C, Heaney J P, Medina M A et al. Storm water management model-user's manual (Version ). U. S. : Environmental Protection Agency, 1987.

[5] Donigian A S Jr, Davis J H. Users manual for agricultural runoff management ( ARM) model. EPA - 600/ 3 - 78 - 080. Environmental Research Laboratory, U. S. EPA, Athens, GA, 1978.

[6] Williams J R, Jones C A, Dyke P T et al. Modeling approach to determining the relationship between erosion and soil productivity. Transactions of the ASAE, 1984, 27(1): 129-144.

[7] Beasley D B, Huggins L F, Monke E J. ANSWERS: A Model for watershed planning. Transactions of the $A S A E, 1980,23(4): 938-944$.

[8] Arnold J G, Srinivasan R, Muttiah T S et al. Large area hydrologic modeling and assessment part: model application. JAWRA, 1998, 43(1): $91-101$.

[9] Smith S J, Kissel D E, Williams J R. Nitrate production, uptake and leaching. In: Creams: A Field scale model for chemical, runoff, and erosion from agricultural management systems. Conservation Research Report USDA-SEA, $1980,26: 493-508$.

[10] 胡雪涛,陈吉宁,张天柱. 非点源污染模型研究. 环境科学, 2002,23(3):124-128.

[11] Amin-Sichani S. Modeling phosphorus transport in surface runoff from agricultural watersheds. PhD Dissertation, Purdue University, West Lafayette, 1982.

[12] Dillaha T A, Heatwole C D et al. Water quality modeling for nonpoint source pollution control planning: Nu- 
trient Transport. Blacksburg, VA: prepared for the Virginia Division of Soil and Water Conservation, Virginia Polytechnic Institute and State University, Dept. of Agricultural Engineering, 1988: 117.

[13] Bouraoui F, Dillaha T A. ANSWER-2000: non-point-source nutrient planning model. Journal of Environmental Engineering, 2000,126(11): 1045 - 1055.

[14] Green W H, Ampt G A. Studies of soil physics, part. The flow of air and water through soils. Journal of Agricultural Science, 1911, 4: $1-24$.

[15] Bouraoui F. Development of a continuous, physically-based, distributed parameter, nonpoint source model. Virginia: Blasksburg, 1994.

[16] Leonard R A, Knisel W G, Still D A. GLEAMS: groundwater loading effects of agricultural management systems. Transactions of the ASAE, 1987, 30(5): $1403-1418$.

[17] Heatwole C T, Dillaha S. Mostaghimi, agricultural BMPs applicable to virginia. Bulletin 169, Virginia Water Resources Research Central, Virginia Polytechnic Institute and State University, Blacksburg, VA, 1991.

[18] Mein R G, Larson C L. Modeling infiltration component of the rainfall-runoff process. Water Resource Research Central Bull. 43. Water Resource research Central, University of Minnesota, Minneapolis, Minnesota, 1971.

[19] Montas H J, Madramootoo C A. Using the ANSWER model to predict runoff and soil Loss in southwestern. Quebec. Transactions of the ASAE, 1991, 34(4) : $1754-1761$.

[20] James L D, Burgess S J. Selection, calibration and testing of hydrologic models. In: Haan C T, Johnson H P \& Brakensiek D L eds. Hydrologic modeling of small watersheds. ASAE, St. Joseph, Mich. , 1982: 437 -472 . 